http://jmscr.igmpublication.org/home/ ISSN (e)-2347-176x ISSN (p) 2455-0450 crossref DOI: https://dx.doi.org/10.18535/jmscr/v8i6.89

\author{
Deurnal Of Medical Science And Clinical Research \\ IGM Publication \\ An official Publication of IGM Publication
}

\title{
Atypical Infantile Kawasaki Disease with Rare Presentation of Purulent Conjuctivitis: Case Report
}

\author{
Authors \\ Nishant Acharya ${ }^{1}$, Priyanka Sharma ${ }^{2}$, Parvinder Singh ${ }^{3}$ \\ ${ }^{1,3}$ Department of Paediatrics, Dr. RKGMC Hamirpur (H.P), India \\ ${ }^{2}$ Department of OBG, Bhandari Hospital Hamirpur (H.P), India \\ *Corresponding Author \\ Priyanka Sharma
}

\begin{abstract}
Kawasaki disease (KD) is a medium vessel vasculitis which presents as an acute febrile illness affecting young children with $80 \%$ affecting less than 5 years and with slight male preponderance. KD remains purely a clinical diagnosis rests on the recognition of a typical temporal sequence of a constellation of clinical features. The main concern about the KD is development of coronary artery abnormalities (CAA) in 15-25\% of affected individuals. The diagnosis of infantile KD can be particularly difficult due to obscure clinical manifestations and presentation is usually incomplete or atypical. Adenovirus poses the greatest diagnostic dilemma which presents with many of the features consistent with the atypical KD. We are illustrating a case of infant with features temporally correlating with Kawasaki disease but some atypical features like purulent conjunctivitis.
\end{abstract}

Keywords: Kawasaki disease, Atypical, Purulent conjunctivitis, Mucocutaneus lymph node syndrome.

\section{Introduction}

Kawasaki disease (KD) was first reported from Japan in 1967 by, Tomisaku Kawasaki at Red cross hospital in Tokyo. He described 50 children who appeared to have a unique set of clinical features which he called the 'Mucocutaneous lymph node syndrome'[1],[2],[3]. Kawasaki disease $(\mathrm{KD})$ is a medium vessel vasculitis which presents as an acute febrile illness affecting young children with $80 \%$ affecting less than 5 years and with slight male preponderance ${ }^{[1],[2],[3]}$. KD remains purely a clinical diagnosis rests on the recognition of a typical temporal sequence of a constellation of clinical features, with none of the features taken individually being of any diagnostic significance whatsoever. Moreover, these clinical features may change from day to day, the spectrum evolves over a period of time i.e 1-3 weeks and the entire clinical spectrum is not seen at any one particular point of time. There is no laboratory test or marker which is pathognomonic of the condition. In the absence of a specific laboratory test for $\mathrm{KD}$, a set of clinical criteria have been established to assist the physician in arriving at a diagnosis ${ }^{[2],[3],[4]}$. (Table 1)

Table 1: Diagnostic criteria for kawasaki disease

1. Fever of at least five days duration.

2. Presence of any four of the following 5 features:

- Changes in extremities (periungual desquamation, indurated edema)

- Polymorphous exanthema(maculopapular rash)

- Bilateral conjuctival injection (non purulent with limbal sparing)

- Changes in the lips and oral cavity

- Cervical lymphadenopathy (unilateral $>1.5 \mathrm{~cm}$ )

3. Exclusion of other diseases with similar findings 
The main concern about the $\mathrm{KD}$ is development of coronary artery abnormalities (CAA) in $15-25 \%$ of affected individuals that may lead to significant long term cardiac morbidity, if not diagnosed and treated in time ${ }^{[1],[2]}$. The differential diagnoses of $\mathrm{KD}$ are viral infections, group A streptococcus infection, Ebstein-barr virus, measles, collegen vascular disorders and drug reactions. When a patient has clinical features not commonly associated with this condition, a diagnosis of "atypical" KD can be made ${ }^{[2],[3],[4]}$. Adenovirus poses the greatest diagnostic dilemma which presents with many of the features consistent with the atypical KD. We are illustrating a case of infant with features temporally correlating with Kawasaki disease but some atypical features like purulent conjunctivitis.

\section{Case Report}

Nine month old infant presented to the outpatient department with complaints of high grade fever for 5 days along with generalized maculopapular rash, irritability and poor oral acceptance. Initial possibility of infective aetiology kept, relevant investigations (Table 2) were sent and started empirically on $3^{\text {rd }}$ generation cephalosporins. In view of persistent fever spikes antibiotics were escalated to meropenum and vancomycin. On day 9 of illness patient developed indurated oedema of dorsal aspect of bilateral hands and feet, angular chelitis (Fig. 2), bilateral purulent conjunctivitis (Fig. 2) and perianal desquamation (Fig 3). Though there were some atypical clinical feature but, Keeping in view the temporal sequence of clinical findings, some suggestive investigations (table 1) and fever not responding to antibiotics possibility of atypical KD was kept. Rapid direct fluorescent antigen test to rule out acute adenoviral infection was done as the clinical feature of purulent conjunctivitis is more commonly Associated with it, which was unremarkable. Intravenous immunoglobulins and aspirin were stared which was followed by prompt resolution of fever spikes. In the second week of illness patient developed periungual desquamation (Fig. 1), leucocytosis, thrombocytosis and suggestive echo with minimal pericardial effusion but with no coronary involvement even on long term follow up of 6 months.

Table 2: Investigation chart

\begin{tabular}{|c|c|c|c|}
\hline S.NO. & INVESTIGATIONS & DAY 5 & $2^{\text {ND }}$ WEEK \\
\hline 1. & Hemoglobin & $8.8 \mathrm{~g} / \mathrm{dl}$ & \\
\hline 2. & Total leucocytes count & $\begin{array}{c}5380 \\
\text { thou/ } / \mu 1\end{array}$ & 21530 thou $/ \mu \mathrm{l}$ \\
\hline 3. & platelets & $\begin{array}{l}180000 \\
\text { thou/ } / \mu 1\end{array}$ & 434000 thou/ $/ \mu 1$ \\
\hline 4. & ESR & $\begin{array}{c}48 \mathrm{~mm}^{\text {st }} \\
\mathrm{hr}\end{array}$ & \\
\hline 5. & CRP & Positive & \\
\hline 6. & Total protein & $6.5 \mathrm{~g} / \mathrm{dl}$ & \\
\hline 7. & Albumin & $3 \mathrm{~g} / \mathrm{dl}$ & \\
\hline 8. & SGOT & $34 \mathrm{u} / \mathrm{l}$ & \\
\hline 9. & SGPT & $22 \mathrm{u} / \mathrm{l}$ & \\
\hline 10. & $\begin{array}{c}\text { Urine } \\
\text { routine/Microscopy }\end{array}$ & Normal & \\
\hline 11. & USG abdomen & Normal & \\
\hline 12. & ECHO & & $\begin{array}{c}\text { Minimal } \\
\text { pericardial } \\
\text { effusion with } \\
\text { normal coronary } \\
\text { arteries. }\end{array}$ \\
\hline
\end{tabular}

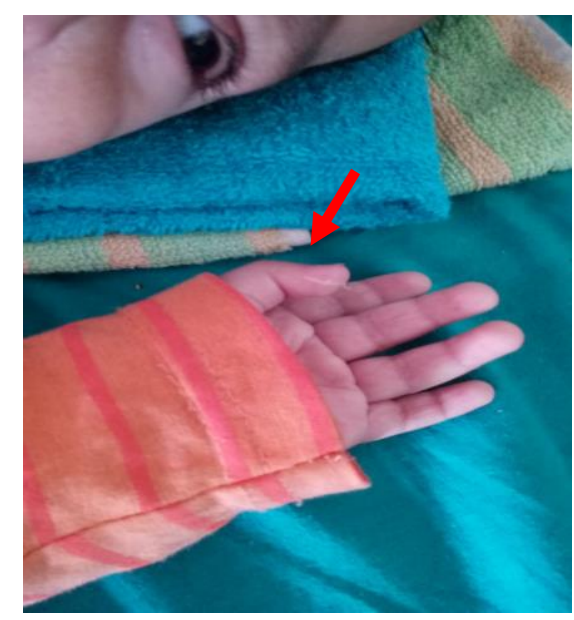

Fig 1 Perungual desquamation

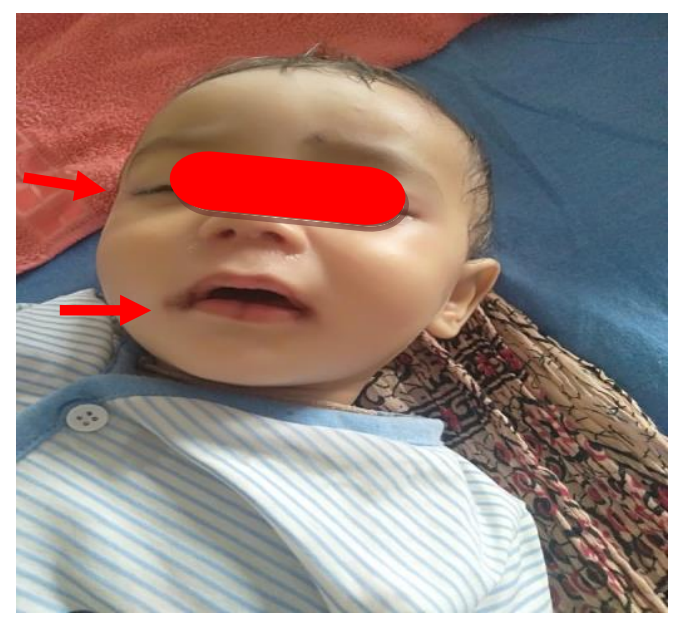

Fig. 2 Angular chilitis with purulent conuctivitis 


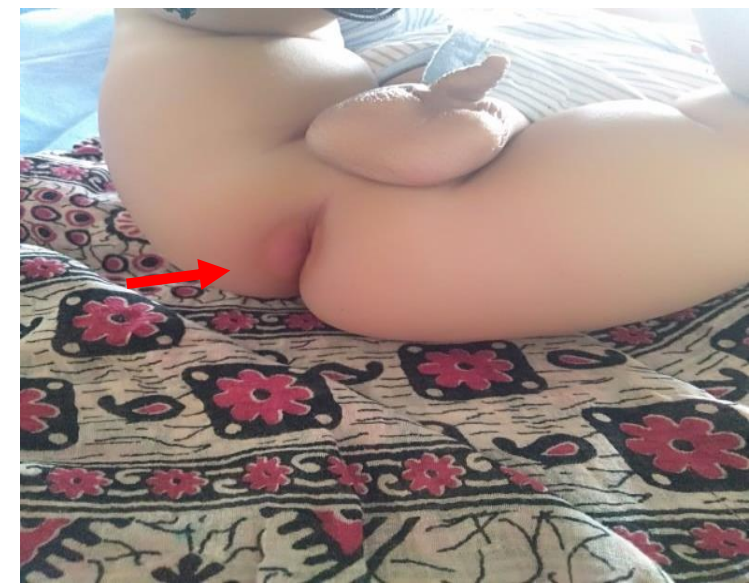

Fig. 3 Perianal desquamation.

\section{Discussion}

Kawasaki disease (KD) is an acute febrile vasculitis. The peak incidence of KD is from 6 months to 2 years of age. $\mathrm{KD}$ is the predominant cause of paediatric acquired heart disease. Cardiac lesions, such as coronary artery aneurysms, are a hallmark of $\mathrm{KD}^{[5],[6]}$. Prompt diagnosis and the administration of intravenous immunoglobulin (IVIG) can reduce the incidence of coronary artery abnormalities from $25 \%$ to $5 \%^{[7]}$. The diagnosis of infantile $\mathrm{KD}$ can be particularly difficult due to obscure clinical manifestations and presentation is usually incomplete or atypical. Some studies have reported that infants younger than 6 months old take the longest time to diagnose, are the least likely to fulfil the major clinical criteria, and have the least favourable laboratory results, all of which are risk factors for developing coronary artery abnormalities [8],[9].

Results of the study done by Shulman et al are consistent. They described $36 \mathrm{KD}$ patients, who were less than 1 year of age during the pre-IVIG era and found that CAA developed in $31 \%$ compared with $18 \%$ in those who were 1 to 2 years of age and $10 \%$ who were more than 2 years of age ${ }^{[10]}$. The clinical challenge lies in distinguishing cases of infantile KD that do not fully meet the diagnostic criteria from those that strongly resemble a variety of common childhood disorders. Thus, have a high index of suspicion should always be kept while evaluating infantile $\mathrm{KD}$, especially under 6months, with unexplained fever for more than 5 days, especially if unresponsive to antibiotics.
Current literature reports that infants below 12 months of age have a higher prevalence of incomplete and atypical $\mathrm{KD}(40 \%)$ compared to older patients $(10-12 \%)^{[11]}$. Our case had a atypical presentation of purulent conjunctivitis. Study done by Stephen $\mathrm{R}$ barone et al demonstrated a statistically significant higher incidence of purulent conjunctivitis in acute adenoviral infection (43\%) as compared to atypical $\mathrm{KD}(8 \%)^{[12]}$, but the latter was ruled out by rapid direct fluorescent antigen assay in our case. Coronary artery aneurysm KD is frequently associated with elevation of inflammatory markers including ESR, CRP, and platelet count. Other laboratory findings such as high white blood cell (WBC) count (neutrophilic type), sterile pyuria, low sodium levels, hypoalbuminemia, or elevated liver enzymes may supplement the diagnosis. Delayed diagnosis and treatment, higher incidence of coronary arteries abnormalities, frequently occur in infantile $\mathrm{KD}$, but thorough appropriate investigations must always be considered along with temporal sequence of a constellation of clinical features to supplement the diagnosis and exclusion of other disease with similar clinical picture to prevent unnecessary administration of immunoglobulin therapy.

\section{Conclusions}

$\mathrm{KD}$ clinical diagnosis below 1 year of age can be very challenging since patients may not have classic signs and symptoms, and individual manifestations may be subtle. Therefore, in young infants with unexplained fever lasting more than 5 days, a clinical possibility of KD must be considered and appropriate investigations performed. An early (<7days) intravenous immunoglobulins must be considered keeping high index of suspicion, after exclusion of the other common childhood diseases with similar manifestations.

\section{References}

1. Kawasaki T. Acute febrile mucocutaneous syndrome with lymphoid involvement with specific desquamation of the fingers and toes 
in children [Japanese]. Arerugi 1967; 16: 178222.

2. Kawasaki T. General review and problems in Kawasaki disease. Jpn Heart J 1995; 36: 1-12.

3. Sundel RP, Petty RE. Kawasaki disease. In: Cassidy JT, Petty RE, Laxer RM, Lindsley CB. Textbook of Pediatric Rheumatology. 5th Ed. Philadelphia: Elsevier Saunders; 2005. p. 521-538.

4. Committee on Rheumatic fever, Endocarditis, and Kawasaki Disease of the American Heart Association's Council on Cardiovascular Disease in the Young. Diagnostic guidelines for Kawasaki disease. Am J Dis Child 1990; 144: 1218-1219.

5. Neuwirth CA, Singh H. Intercostal artery aneurysm in a child with Kawasaki disease and known coronary artery aneurysms. J Vasc Interv Radiol 2010; 21: 952-3.

6. JCS Joint Working Group. Guidelines for diagnosis and management of cardiovascular sequelae in Kawasaki disease (JCS 2008): digest version. Circ J 2010; 74: 1989-2020.

7. Kim SH, Kim KH, Kim DS. Clinical characteristics of Kawasaki disease according to age at diagnosis. Indian Pediatr 2009; 46: 585-90.

8. Burns JC, Wiggins JW Jr, Toews WH, Newburger JW, Leung DY, Wilson H, et al. Clinical spectrum of Kawasaki disease in infants younger than 6 months of age. J Pediatr 1986; 109: 759-63.

9. Liu HC, Lo CW, Hwang B, Lee PC. Clinical manifestations vary with different age spectrums in infants with Kawasaki disease. Scientific World Journal 2012; 2012: 210382.

10. Shulman ST, McAuley JB, Pachman LM, Miller ML, Ruschhaupt DG. Risk of coronary abnormalities due to Kawasaki disease in urban area with small Asian population. Am J Dis Child. 1987; 141: $420-5$.
11. Sundel RP, Petty RE. Kawasaki disease. In: Cassidy JT, Petty RE, Laxer RM, Lindsley $\mathrm{CB}$, editors. Textbook of pediatric rheumatology. 6th ed. Philadelphia: Elsevier Saunders; 2011. p. 505-20.

12. Stephen $\mathrm{R}$ barone et al. The differentiation of classical Kawasaki disease, atypical Kawasaki disease, and acute Adenoviral infection. Arch pediatr adoles med 2000; 154: 453-56. 